TRANS · núm. 25.2021

MISCELÁNEA · 459-473

CIENTIFFICA

Las características y el sector de la traducción farmacéutica obligan a los traductores a enfrentarse al dilema de a qué variedad diatópica del español traducir. La práctica diaria exige a los traductores farmacéuticos traducir frecuentemente a variedades de las que no son nativos, eminentemente al español peninsular, al español latinoamericano/hispanoamericano, al español neutro y a otras variedades locales dentro de Hispanoamérica. Este artículo tiene como fin efectuar una reflexión académica sobre el uso de variedades del español de los encargos de traducción de textos farmacéuticos, con especial énfasis en el español neutro, con base en la experiencia profesional del autor.

PALABRAS CLAVE: traducción farmacéutica, variedades diatópicas, español neutro, español peninsular, español hispanoamericano, español latinoamericano.

\title{
Variedades diatópicas del español y español neutro en traducción farmacéutica: una reflexión académica desde una perspectiva profesional
}

\author{
Miguel Ángel Cascales Serrano \\ Universidad de Málaga
}

\section{Diatopic Varieties of Spanish and Neutral Spanish in Pharmaceutical Translations: an academic reflection from a professional perspective}

The characteristics and the industry of pharmaceutical translations force translators to face the dilemma of which diatopic variety of Spanish to use when translating. The daily practice of pharmaceutical translators often requires them to translate into varieties other than their native tongue, mainly into Peninsular Spanish, Latin-American/Hispanic-American Spanish or neutral Spanish, as well as into other local varieties in Hispanic America. This paper aims at carrying out an academic reflection on the use of Spanish varieties in translation assignments of pharmaceutical texts, with particular emphasis on neutral Spanish, based on the author's professional experience.

KEY WORDS: pharmaceutical translation, diatopic varieties of Spanish, neutral Spanish, Peninsular Spanish, Hispanic-American Spanish, LatinAmerican Spanish. 


\section{1. INTRODUCCIÓN}

Desde el prisma del traductor de textos farmacéuticos, la industria farmacéutica destaca eminentemente por dos notas características. Por un lado, su importante componente de globalización, derivado del alcance general de los productos que suministra. Por otro, su celeridad, fruto del constante e infatigable cambio y control que se efectúa en dichos productos o en la creación de otros que satisfagan necesidades aún por cubrir.

Dichas características, la globalización y la celeridad, impregnan el proceso de traducción de textos farmacéuticos, entendido como el de la traducción de toda aquella documentación que rodea la industria farmacéutica (investigación, aprobación de medicamentos/productos sanitarios, comercialización, farmacovigilancia, fabricación, etc.). La globalización se refleja en la constante necesidad de traducir documentación para su uso en múltiples países y a ambos lados del Atlántico. A su vez, la celeridad se concreta en el requisito, frecuentemente dispuesto por ley, de traducir un número elevado de textos - en general, de gran extensión - en los reducidos plazos que imponen tanto la tramitación de solicitudes de aprobación o comercialización de medicamentos y productos sanitarios como las necesidades técnicas y estructurales de las empresas del sector. Sumados estos factores al alto grado de especialización que requiere la traducción de este tipo de textos, es frecuente que las empresas farmacéuticas - $-\mathrm{y}$, por extensión, las empresas de traducción que las asisten- tengan dificultades para encontrar a traductores de la zona geográfica a la que está destinado el texto traducido dentro de los plazos pertinentes y, en consecuencia, tengan que recurrir a traductores «no nativos».

Es por ello que la práctica diaria de la traducción farmacéutica demuestra que no es infrecuente que se requiera traducir para España ylos países de Hispanoamérica indistintamente de la variedad vernácula del traductor, generalmente con un proceso de revisión posterior por parte de un traductor nativo de la región. Incluso aquellas empresas que únicamente trabajan con traductores nativos de la variedad diatópica meta - suelen ser empresas no especializadas únicamente en traducción médica/farmacéutica con un volumen de traducción farmacéutica que les permite encontrar traductores a tiempo- recurren en última instancia a traductores de zonas geográficas distintas cuando el tiempo apremia.

Asimismo, la globalización suele implicar que las traducciones farmacéuticas tengan como destino múltiples países de habla hispana o se dirijan a la generalidad de los hispanohablantes, así como la celeridad hace que, en la mayoría de los casos, no sea posible efectuar una localización a las variedades de zonas geográficas concretas dada la limitación de los plazos. Es aquí donde entra en juego el papel del denominado español neutro. La traducción a la variedad neutra del castellano destaca como requisito de traducción frecuentemente explicitado por los clientes o implícitamente autoimpuesto por los traductores a falta de directrices precisas por parte de las empresas que solicitan dichos servicios de traducción.

Sobre el papel y sin mayor reflexión, los términos español peninsular, español neutro, español latinoamericano o español hispanoamericano parecen, en mayor o menor medida, claros en la cabeza de los profesionales de textos especializados, como es el caso de la traducción farmacéutica. Sin embargo, bajo una lupa de mayor aumento, cabe realizar una reflexión más profunda: ¿a qué se refiere un cliente cuando requiere traducir al español neutro? ¿Dónde se sitúa el límite de la neutralidad? ¿Es posible la neutralidad en traducción farmacéutica o, si cabe, en otros tipos de traducción? ¿Qué es traducir al español hispanoamericano? ¿Es diferente a traducir al español latinoamericano? Y, 
en consecuencia, ¿existe un lenguaje farmacéutico común para la totalidad de hispanoamericanos distinto al lenguaje farmacéutico peninsular? ¿Garantizan las cadenas de traducción actuales la traducción a la variedad escogida? El presente artículo tiene como objetivo reflexionar en torno a estas cuestiones mediante un análisis de aspectos pragmáticos fundamentales con base en la experiencia del autor como traductor farmacéutico, con el objeto de que dicha reflexión constituya un punto de partida para futuras investigaciones centradas en aspectos teóricos.

\section{LA LEGISLACIÓN NACIONAL COMO DE- TERMINANTE DE LA VARIEDAD EN TRA- DUCCIÓN FARMACÉUTICA}

Antes de entrar en materia, es preciso señalar que la traducción farmacéutica destaca por ser uno de los ámbitos de traducción especializada con una reglamentación más rigurosa en cuanto al formato y al lenguaje empleados en los textos. Dicha reglamentación emana del interés público de claridad y univocidad, características, como se verá, de los lenguajes de especialidad, cuya carencia puede acarrear consecuencias para la salud de los usuarios. Así, el proceso de aprobación y vigilancia de medicamentos y productos sanitarios está tasado legalmente y así lo está el lenguaje de los documentos presentados a las autoridades a lo largo de dicho proceso.

Sin proceder aquí a realizar un recorrido por las distintas obligaciones legales en cuanto al uso del español en textos farmacéuticos, la tendencia general es que cuanto más próximo esté el texto al usuario final, es decir, al paciente o al profesional sanitario, más pautados están legalmente tanto el formato del documento como su contenido — redacción, apartados, terminología, registro, etc.- - Así, en la cúspide de la reglamentación, se encuentran los prospectos (destinados a los pacientes) y las fichas técnicas o resúmenes de las características del producto (destinados a los profesionales sanitarios). Para dichos documentos, al menos en el ámbito europeo, deben seguirse estrictamente las plantillas obligatorias dispuestas por las autoridades, con formato, terminología y expresiones estandarizadas, so pena de ver rechazada la aprobación de un medicamento por causas imputables a la traducción (EMA, 2021a).

Asimismo, para todos aquellos documentos presentados ante la Agencia Europea de Medicamentos (EMA), la Agencia Española de Medicamentos y Productos Sanitarios (AEMPS) y la mayoría de autoridades de países de Hispanoamérica, es recomendable $-\mathrm{y}$ así se suele requerir en los encargos de traducción- seguir la terminología relativa a afecciones del Medical Dictionary for Regulatory Activities (MedDRA) y, en casos precisos, resulta obligatorio respetar la terminología relativa a las formas farmacéuticas, vías/ métodos de administración, recipientes y otros términos técnicos aprobada en cada región (p. ej., en Europa, la aprobada por el Directorio Europeo para la Calidad de los Medicamentos y de los Servicios Sanitarios [EDQM]) (EDQM, 2021).

Por tanto, es indispensable para el traductor conocer — por sí mismo y por medio de los glosarios, las memorias de traducción y demás herramientas aportadas por los responsables de los proyectos de traducción- la terminología oficial del país destinatario para que la traducción resulte aceptable no solo desde el punto de vista conceptual, sino también en el plano administrativo y jurídico. Se erige así la legislación nacional en materia de productos farmacéuticos de los distintos países hispanohablantes como determinante de la variedad en traducción farmacéutica, puesto que los lenguajes especializados, al igual que el lenguaje común, no están exentos de la existencia de variaciones diatópicas (García Izquierdo, 2006a). 


\section{3. VARIEDADES DEL ESPAÑOL EN TRADUC- CIÓN FARMACÉUTICA}

Con el fin de acotar el objeto de estudio, parece pertinente aportar una definición de los términos español peninsular, español neutro, español latinoamericano y español hispanoamericano, sin olvidar que la delimitación de las variedades diatópicas de una lengua siempre resulta problemática, ya que implica la existencia de «cierta conciencia lingüística por parte de sus hablantes sobre la autonomía diferencial de su variedad» (Fernández-Ordoñez, 2016).

\subsection{El español peninsular}

El español de España, español ibérico, español peninsular o español europeo debe entenderse como la lengua estándar de España, comprensible y utilizada por la generalidad de los españoles. Precisamente el término español peninsular, que es el más empleado en encargos de traducción junto al de español (España), puede resultar poco acertado, dado que denotativamente se refiere exclusivamente al usado en la Península y excluye así a las Islas Canarias, las Islas Baleares, Ceuta y Melilla, territorios que, no obstante, comparten lengua estándar con el resto de España. Realizada esta matización, es preciso indicar que, en el ámbito de la traducción farmacéutica, no parece pertinente analizar las divisiones dialectales del español de España por diversas razones.

En primer lugar, porque, al contrario que con otros tipos de traducción (especialmente la literaria), no resulta deseable que dichas variedades dialectales se viertan en las traducciones de textos farmacéuticos. Esto se debe a que es obligación de conformidad con la legislación de la Unión Europea que exista una única versión de distintos textos (prospectos, resúmenes de las características del producto, etiquetado, entre otros) por lengua de los Estados miembros
(AEMPS, 2010). En el caso de España, dichas traducciones deben ser aprobadas por la autoridad competente en la materia, es decir, la Agencia Española de Medicamentos y Productos Sanitarios (AEMPS). En consecuencia, dichos textos tienen como público objetivo en primera instancia a la AEMPS (sede sita en Madrid) y posteriormente al conjunto de los españoles.

En segundo lugar, porque existen ciertos determinantes legislativos en el plano terminológico que impiden emplear léxico propio de una variedad dialectal regional de España. Por un lado, es obligatorio emplear para las formas farmacéuticas el listado de términos estándar (standard terms) de la Dirección Europea de la Calidad de los Medicamentos y la Asistencia Sanitaria (EDQM) del Consejo de Europa (AEMPS, 2016). Por otro lado, como se ha mencionado, existe la obligación general en la Unión Europea de emplear los términos médicos (signos, síntomas, enfermedades, indicaciones terapéuticas, procedimientos, órganos y aparatos, etc.) del Diccionario médico para actividades de registro farmacéutico (MedDRA), publicado por el Consejo Internacional de Armonización (ICH). Esto es así porque dicho ICH se creó en 1996 para facilitar la estandarización y codificación internacional de la terminología relativa a los efectos adversos (Marcilly et al., 2020) y la Unión Europea, con la Agencia Europea del Medicamento (EMA) como autoridad competente, recalca el uso de este diccionario como requisito para las actividades de registro con el fin de estandarizar la terminología y evitar conversiones terminológicas que den lugar a distorsiones de datos (EMA, 2021b).

Por último, y en consecuencia con lo anterior, es patente que las traducciones deben ser comprendidas inequívocamente y de la forma más unívoca posible por el conjunto de los españoles por evidentes razones sanitarias de interés público. Existiendo esa obligación de comprensión generalizada, cualquier marca dialectal regional 
no solo puede constituir un obstáculo para dicho requisito, sino que puede suponer el rechazo de la traducción por parte de la autoridad de registro farmacéutico competente por no cumplir el principio de comprensión (AEMPS, 2016).

\subsection{El español latinoamericano/hispanoa- mericano}

Más problemáticos resultan los términos espanol latinoamericano y español hispanoamericano, e incluso español iberoamericano, dejando a un lado otros como sudamericano/suramericano, centroamericano, hispanounidense $\mathrm{u}$ otros por no representar la variedad del conjunto de los hispanohablantes de América, en caso de que dicha variedad realmente exista para el objeto del presente estudio.

No parece suficiente en la práctica de la traducción farmacéutica la distinción recomendada por el Diccionario panhispánico de dudas (RAE, 2005) en cuanto a los gentilicios hispanoamericano, latinoamericano e iberoamericano, que, aplicada a las lenguas, serviría para distinguir al español hispanoamericano como la variedad propia del «conjunto de países americanos de lengua española», al español latinoamericano como aquella perteneciente al «conjunto de países del continente americano en los que se hablan lenguas derivadas del latín (español, portugués y francés)» y al español iberoamericano como la de «los países americanos de lengua iberorromance (portugués y español)» (FUNDEU, 2011). De estas divisiones conceptuales cabría concluir que el término idóneo para referirse únicamente al español de los países hispanohablantes de América es hispanoamericano. No obstante, la práctica diaria demuestra que, al recibir un encargo de traducción farmacéutica, en raras ocasiones se emplea dicho término y lo más corriente es que se indique que debe hacerse al español latinoamericano (LATAM), denominación, por otro lado, preferida mayoritariamente por los hispanohablantes de América de acuerdo con 463 una encuesta (Soca, 2010; La Prensa; 2010).

Efectuada esta matización, parece pertinente cuestionarse si cabe hablar de un español latinoamericano en traducción farmacéutica, es decir, de una lengua estándar empleada y comprendida por la generalidad de los hispanohablantes de Latinoamérica, al igual que con el análisis efectuado en el apartado anterior para el español peninsular. Llegados a este punto, procede presentar varios argumentos a favor y en contra de dicha hipótesis.

A favor, es posible argüir que el lenguaje farmacéutico - y el médico por extensión- del español empleado por los distintos países de Latinoamérica comparte características comúnmente aceptadas. Así, es una característica compartida - si bien en muchos casos no una obligación como en la UE- que todos sigan el citado MedDRA del ICH, que, recordemos, también respeta España. De hecho, EE. UU. es miembro del ICH y Argentina, Cuba, México y Colombia son observadores de dicho consejo (ICH, 2020).

Por otro lado, existen tendencias lingüísticas y términos generalmente compartidos entre dichos países que difieren sustancialmente de la variedad de España. A modo de ejemplo, tómese el vocablo inglés adverse event, esencial en traducción farmacéutica, que denomina a «cualquier experiencia médica imprevista o indeseable que ocurra a un sujeto durante un ensayo clínico, se considere o no relacionada con el tratamiento en estudio». Dicho término se traduce en Latinoamérica como evento adverso (EA) y en España como acontecimiento adverso (AA) (A. Navarro, 2020), si bien también se usa el término hispanoamericano, aunque con menor prevalencia. Asimismo, el término sponsor suele traducirse en Latinoamérica como patrocinador (o patrocinante en Chile), mientras que en España se emplea promotor de un ensayo clínico. Es más, el uso de patrocinador en España está firmemente desaconsejado por parte de los clientes e incluso hay traductores a los que 
464 les resulta «sospechoso», por suscitar menor objetividad en el lector (Rodríguez-Perdomo, 2012).

En contra del hipotético español latinoamericano como lengua estándar en traducción farmacéutica, hay que subrayar que la situación de la traducción farmacéutica en Latinoamérica es bien distinta a la de España, así como bastante más compleja. Dicha complejidad emana de la descentralización existente en Latinoamérica, dado que, al contrario que con la EMA en la UE, no existe una agencia reguladora supranacional para los 19 países hispanoamericanos. En consecuencia, hay una multiplicidad de agencias reguladoras a las que debe solicitarse el registro $u$ otro tipo de trámite relativo a productos farmacéuticos, cada una con sus requisitos de forma, procedimiento y diferencias terminológicas.

A efectos ilustrativos, se muestra a continuación una lista que refleja la citada multiplicidad de autoridades competentes:

Tabla 1. Lista de autoridades reguladoras de países hispanohablantes que forman parte de la Red de Autoridades en Medicamentos de Iberoamérica (Red EAMI, n. d.), a la que se añade Puerto Rico (EE. UU.), que no forma parte de dicha Red

\begin{tabular}{|c|c|}
\hline País & Autoridad reguladora \\
\hline Argentina & Administración Nacional de Medicamentos, Alimentos y Tecnología Médica -ANMAT \\
\hline Bolivia & Agencia Estatal de Medicamentos y Tecnologías en Salud -AGEMED \\
\hline Chile & Agencia Nacional de Medicamentos -ANAMED- Instituto de Salud Pública -ISP \\
\hline Colombia & Instituto Nacional de Vigilancia de Medicamentos y Alimentos -INVIMA \\
\hline Costa Rica & Dirección de Regulación de Productos de Interés Sanitario del Ministerio de Salud \\
\hline Cuba & Centro para el Control Estatal de Medicamentos, Equipos y Dispositivos Médicos-CECMED \\
\hline Ecuador & Agencia Nacional de Regulación, Control y Vigilancia Sanitaria -ARCSA \\
\hline El Salvador & Dirección Nacional de Medicamentos -DNM \\
\hline Guatemala & Departamento de Regulación y Control de Productos Farmacéuticos y Afines -DRCPFA \\
\hline Honduras & Agencia de Regulación Sanitaria-ARSA \\
\hline México & Comisión Federal para la protección contra Riesgos Sanitarios -COFEPRIS \\
\hline Nicaragua & Dirección General de Regulación Sanitaria-Ministerio de Salud \\
\hline Panamá & Dirección Nacional de Farmacia y Drogas \\
\hline Paraguay & Dirección Nacional de Vigilancia Sanitaria -DINAVISA \\
\hline Perú & Dirección General de Medicamentos, Insumos y Drogas -DIGEMID \\
\hline $\begin{array}{l}\text { Puerto Rico } \\
\text { (EE. UU.) }\end{array}$ & Administración de Medicamentos y Alimentos (Food and Drug Administration) -FDA \\
\hline $\begin{array}{l}\text { República } \\
\text { Dominicana }\end{array}$ & Dirección General de Medicamentos, Alimentos y Productos Sanitarios -DIGEMAPS \\
\hline Uruguay & División Evaluación Sanitaria - Ministerio de Salud Pública-MSP \\
\hline Venezuela & Instituto Nacional de Higiene Rafael Rangel-INHRR \\
\hline
\end{tabular}


Como ejemplo de esta multiplicidad de autoridades reguladoras que origina importantes diferencias terminológicas entre los países latinoamericanos, tomemos el caso del vocablo inglés medical device, término también central en traducción farmacéutica. Este término, que se traduce obligatoriamente como producto sanitario en España, da nombre según el Libro rojo de Fernando A. Navarro a «cualquier artículo médico o sanitario utilizado con fines diagnósticos, terapéuticos o profilácticos que no actúe en el cuerpo humano a través de un mecanismo químico, farmacológico, inmunitario o metabólico» (A. Navarro, 2020). De acuerdo con este mismo diccionario, la traducción oficial del término medical device varía de país a país de conformidad con la legislación nacional en cuestión. Así, «en la Argentina se usa "producto médico"; en Méjico [sic], "equipo médico" o "dispositivo médico", según el contexto; en Chile, "dispositivo de uso médico” o “dispositivo médico”, y en Venezuela, “dispositivo médico”» (A. Navarro, 2020).

Por otro lado, al haber distintas agencias reguladoras, la denominación de los productos farmacéuticos suele contener cambios entre los distintos países, a veces de carácter muy marcado con nombres que no son en absoluto similares. A modo de ejemplo, el fármaco con denominación común internacional paracetamol, se denomina en Puerto Rico acetaminofén (A. Navarro, 2020). Conviene así no entrar en el ámbito de los nombres comerciales - no confundir con las denominaciones comunes internacionales-, donde la variopinta disparidad de maneras de llamar a un mismo medicamento - en ocasiones con diferencias muy sutiles - trae de cabeza a los traductores y merece un estudio aparte.

Es necesario volver a incidir en que estas diferencias terminológicas no son meramente anecdóticas, sino que implican conceptos con consecuencias para la salud de las personas y los animales, y, por ende, el uso de un término 465 incorrecto con arreglo a la legislación de un país puede generar problemas administrativos importantes y, en esencia, dar lugar al rechazo de las traducciones presentadas.

Vistos los argumentos a favor y en contra, parece aventurado afirmar que exista un único español farmacéutico latinoamericano y más prudente aseverar lo contrario, si bien se evidencian elementos lingüísticos comunes que distancian al/a los lenguaje/s farmacéutico/s latinoamericano/s del peninsular. No obstante, a pesar de la existencia de diferencias terminológicas relevantes, debe apuntarse que la realidad de la traducción farmacéutica, como la del resto de especialidades, demuestra que coexisten en el sector prácticas más cuidadosas y otras menos atentas con la preservación de la variedad diatópica meta. Lo más frecuente es que, si el texto tiene como destino Latinoamérica, se indique en las especificaciones del proyecto dicha circunstancia, junto con el país específico al que va destinado. Dicho esto, desafortunadamente, no son práctica infrecuente las cadenas de traducción en las que no se especifica el país de destino, por lo que no es extraño que se traduzca a un español latinoamericano general y se obvien las diferencias dialectales que pudieren presentarse, con los posibles problemas administrativos que ello acarrea.

\subsection{El español neutro}

Si resulta conflictivo afirmar y acotar la existencia de un español peninsular y un español latinoamericano como lenguas estándar en un lenguaje especializado como el de la traducción farmacéutica, la dificultad de delimitación se acrecienta llegados al español neutro. En esencia, se trata de afirmar o descartar la existencia de un español que permita la comunicación universal y unívoca entre todos los hispanohablantes den- 
466 tro del ámbito farmacéutico: según la descripción de Ramírez Luengo (2011), una lingua franca fruto de la koneización dirigida ${ }^{1}$.

Antes de proseguir, sería adecuado tener presente que procedemos a analizar la posible existencia del español neutro en un lenguaje especializado. Como aclara Cabré (2004), los lenguajes especializados son «registros funcionales caracterizados por una temática específica [...] conceptualizada de manera específica [...] en situaciones de comunicación en las que el emisor o los emisores son específicos [...], el tipo de situación también lo es [...], y la función comunicativa que les es inherente es la informativa». Los lenguajes de especialidad tienen como características esenciales el ser denotativos - justamente para priorizar esa función comunicativa informativa-, tener tendencia a la universalidad y aspirar a la univocidad con la voluntad de concisión y precisión (García Izquierdo, 2006a).

Por consiguiente, dentro del marco de los lenguajes de especialidad, que tienden a adoptar un papel eminentemente unificador, el objetivo de un posible español neutro es sencillamente el de la comprensión mutua (Sáenz, 2013). Esta nota esencial se contrapone a la de otros ámbitos de traducción como el de la traducción literaria o audiovisual, en los que el uso de las distintas variedades diatópicas del español (incluida la del posible español neutro) parece poseer mayor relevancia y puede llegar a determinar la aceptación o el rechazo de las traducciones (Gargatagli, 2012; Villalba, 2017; Zaro Vera, 2013). En consecuencia, no se dan aquí - o se dan en mucha menor medida - las reivindicaciones lingüísticas identitarias que rechazan el español neutro

I Convergencia progresiva y consciente de las variedades sin nativización de la koiné resultante, al contrario que con la koneización no dirigida, en la que el proceso de koneización puede acabar con la nativización de la variedad final (Ramírez Luengo, 2011: 18-20). en traducción literaria por ser una variedad artificial que no posee la riqueza lingüística que aporta el uso de las variedades regionales. Por ello, hay autores que han optado por acuñar una denominación específica para el español neutro empleado dentro de los lenguajes de especialidad. Así, el español neutro en dicho ámbito se ha llamado «español técnico neutro» (Castro, 2017), «internacional» (Gómez Font, 2012; Bravo García, 2008) o «culto» (Sáenz, 2013), entre otras denominaciones atribuidas al fenómeno.

Si enmarcamos la traducción farmacéutica como lenguaje de especialidad y partimos de la existencia de los componentes que rigen su práctica diaria —a saber, la globalización y la celeridad-, la existencia de un español neutro en este marco parecería bastante pertinente y deseable. Lógicamente, traducir un informe de un ensayo clínico de cientos de páginas una única vez sin necesidad de localización o adaptación posterior conllevaría un ahorro importante en términos económicos, logísticos y, sobre todo, de tiempo, lo que permitiría cumplir con los ajustados plazos más fácilmente $y$, a la par, posibilitaría reducir los gastos incurridos en traducción para la internacionalización de los productos farmacéuticos. Por estas razones tan convenientes, hay autores que se han inclinado en la dirección de la defensa del español neutro dentro de los lenguajes de especialidad (García Izquierdo, 2006a, 2006b). De hecho, hay empresas farmacéuticas y de traducción que remiten artículos en defensa del español neutro junto con los paquetes de los encargos, quizás con el objeto de «concienciar» a los traductores sobre a qué variedad deben aspirar a traducir en determinados casos.

No obstante, se evidencia en estos intentos por instaurar definitivamente el español neutro en la traducción farmacéutica - en ocasiones, a toda costa - una carencia de reflexión honda sobre las características actuales y reales del len- 
guaje farmacéutico en nuestra lengua. Dicha falta de mayor reflexión no es nimia, dado que, haciéndoles pensar que el español en este ámbito es uno y que cuando se habla de algo todos queremos decir lo mismo, se puede llegar a confundir profundamente a los traductores $y$, en última instancia y a través de estos, a los profesionales sanitarios, a los investigadores y a los pacientes.

\subsubsection{Unificación en el plano léxico}

A pesar de que la notable homogeneidad léxica del español ha quedado demostrada en diversos estudios lexicoestadísticos (López Morales, 2006, 172-194), es interesante reflexionar sobre los procesos o herramientas de unificación léxica en un lenguaje especializado como el farmacéutico, teniendo en cuenta que quizás el plano léxico es, junto al textual, el más relevante en dicho tipo de lenguajes (Cabré y Estopá, 2005). Efectivamente, muchos autores, entre ellas Cabré, han coincidido en que las lenguas de especialidad «disponen de reglas establecidas voluntariamente por los usuarios y aceptadas a nivel internacional, lo que facilita la intercomprensión entre los usuarios de las mismas» (García Izquierdo, 2006a). En este punto, cabe traer a colación dos de las herramientas principales dentro del ámbito médico y farmacéutico que aspiran a dicha universalidad terminológica para analizarlas en mayor profundidad. Por una parte, el mencionado MedDRA del ICH, un diccionario que busca la internacionalización de cierta terminología médica. Por otra, las también citadas denominaciones comunes internacionales (DCI; international nonpropietary name o INN, en inglés), propuestas por la Organización Mundial de la Salud, para los nombres oficiales no comerciales o genéricos de las sustancias farmacológicas.

Desafortunadamente, dos obstáculos fundamentales se interponen entre estas dos he- rramientas esenciales y la internacionalización 467 terminológica.

Por un lado, cuando hablamos de traducción farmacéutica, hacemos referencia eminentemente al ámbito de las ciencias experimentales. $\mathrm{Si}$ algo caracteriza a la industria farmacéutica es la constante innovación. La celeridad demandada a las traducciones encuentra su base en dicho cambio infatigable: las empresas luchan entre sí por mejorar el producto de la competencia a un ritmo frenético. Lo que ayer era el medicamento que mejor cubría una necesidad médica hoy queda desterrado al olvido. Cualquier profesional de la traducción farmacéutica es testigo privilegiado —cláusula de confidencialidad de por mediode que nada en el sector farmacéutico es estático. No hay mejor prueba que la que nos ofrece la actualidad: véase la feroz competencia farmacéutica por encontrar la vacuna para la COVID-19.

En este caldo de cultivo, surgen constantemente neologismos para describir conceptos nuevos que un diccionario, por ambiciosa que sea su aspiración de internacionalización terminológica, no puede abarcar. Es principalmente a los investigadores y a los traductores a los que les toca en primera instancia decidir cómo se va denominar algo en una lengua determinada. Por ello, en este entramado de competencia e innovación, prima la celeridad y, en consecuencia, la necesidad de unificación terminológica, que toma tiempo, queda relegada a un segundo plano. Incluso aquellos que defienden la necesidad del español técnico neutro reconocen que las ciencias experimentales «tienen características específicas (aumento significativo de géneros divulgativos, terminología poco homogénea, etc.) que las hacen especialmente sensibles a este intento de homogeneización lingüística» (García Izquierdo, 2006a).

Por otro lado, el MedDRA y las DCI carecen, en su afán de internacionalización, precisamente 
468 de lo que prometen: de la suficiente aceptación internacional.

Así, el uso del MedDRA es solo obligatorio en ciertos territorios (p. ej., en la Unión Europea), por lo que su implantación en otros territorios, más allá de constituir una recomendación general (especialmente en Latinoamérica), puede considerarse ciertamente deficiente (Marcilly, 2020). Es más, en aquellos territorios en los que existe la obligación de emplear la terminología del diccionario, es permisible cierto grado de desviación de la terminología que el diccionario recoge al traducir. Así ocurre en la práctica diaria, en la que las empresas farmacéuticas y agencias de traducción, en la opinión de que la calidad de las traducciones del diccionario es mejorable, suelen alentar explícitamente al traductor en las especificaciones del encargo a optar por una traducción distinta a la del MedDRA, debiendo aportar, eso sí, los motivos que le han llevado a ello.

Lo mismo ocurre con las DCI. Su uso es amplio y mayoritario, pero hay territorios que cuentan con denominaciones propias para las sustancias farmacológicas. Por ejemplo, en Puerto Rico se usan los United States Adopted Names (USAN), que, como se ha visto, pueden variar sustancialmente con respecto a las DCI.

Llegados a este punto, teniendo en cuenta que las dos herramientas principales de unificación terminológica no consiguen la absoluta internacionalización y, como se ha constatado en los dos subapartados anteriores, existen diferencias patentes entre las terminologías oficiales de los distintos países hispanohablantes - y, de forma más marcada, entre España y Latinoamérica-, quizás resulte aventurado afirmar que existe una unificación terminológica suficiente en español como para hablar de la existencia de un español neutro en traducción farmacéutica.

Es indudable la tendencia a la unificación del español en este lenguaje de especialidad. No solo por el impulso que aportan las citadas herramientas, sino por parte de los responsables de los proyectos de traducción, cada vez más conscientes de que realmente conviene converger a una única lengua por las ventajas que ello aporta.

Es tanto lo que hay en juego en la industria farmacéutica - gastos desorbitados en traducción y pérdidas aún más desorbitadas en problemas administrativos que retrasen la comercialización de un producto- que lejos quedan aquellos clientes que encargaban grandes proyectos multinacionales y no proveían a sus traductores de glosarios, guías de estilo y memorias de traducción. Hace más de veinte años que algunos autores, Xosé Castro entre ellos en 1996 (2017), aseveraban acertadamente que la concienciación de los responsables de los proyectos sobre la elaboración de estas herramientas traductológicas era esencial para fomentar la ampliación del mercado de la traducción y evitar la disgregación de la terminología en español (García Izquierdo, 2006a). De hecho, en la actualidad existen grandes empresas especializadas únicamente en traducción médico-farmacéutica, lo que evidencia esa creciente concienciación en cuanto a las características específicas de este tipo de traducciones, en las que un error puede costar caro. Por supuesto, no quiere afirmarse con esto que no existan empresas y agencias de traducción médica/farmacéutica que no faciliten dichas herramientas a sus traductores, sino que la práctica diaria demuestra que la actitud y la tendencia contrarias son claramente mayoritarias.

Con estos avances en herramientas terminológicas y en concienciación sobre las diferencias lingüísticas existentes entre España y los distintos países de Latinoamérica, es posible que el lenguaje farmacéutico esté tendiendo progresivamente hacia el ideal de español neutro en el plano léxico. No obstante, al no existir unificación en cuanto a la terminología oficial según las 
distintas legislaciones, el paso siguiente es claro si se tiene el español neutro como meta: para aproximarse a una terminología farmacéutica universal en español es fundamental el consenso. ¿Cómo se cede en este contexto? ¿Quién cede primero? ¿España en dejar de utilizar obligatoriamente producto sanitario o Argentina en usar producto médico? Es más, cabría preguntarse, ¿tendrían ambos que ceder en pos de alcanzar la entelequia del español neutro?

\subsubsection{El español neutro como lengua pivote en la cadena de traducción}

Ocurre que el español neutro dentro de la cadena de traducción se emplea como producto final, pero también es solicitado por empresas y agencias de traducción como producto intermedio, como una suerte de lengua pivote, si se efectúa el símil con la interpretación indirecta o por relé. Así, se requiere frecuentemente traducir a un español neutro para su posterior adaptación a un territorio determinado por parte de un traductor nativo.

Este denominado español neutro como paso intermedio plantea varios problemas en su supuesta neutralidad de la manera en la que suele formularse por parte de los responsables de los proyectos. Para comprender dichos problemas, resulta conveniente tener en mente la cadena de traducción en su conjunto (veáse la Tabla 2).

El primer escollo para la neutralidad es el condicionamiento del traductor/poseditor, que generalmente sabe de antemano cuál será la variedad final y, por tanto, se esfuerza en que el texto tienda más a dicha variedad que a la neutralidad, desde una óptica lógica de optimizar la productividad dentro de la cadena de traducción para ahorrar esfuerzo posterior al revisor y al responsable del control de calidad o posproducción. En este mismo sentido, el traductor/ poseditor conocedor de que el texto tiene como público objetivo un lector hispanoamericano o español está obligado en ciertos casos a respetar la terminología de la región meta.

El segundo problema es que el cliente o la agencia suele predeterminar a través de glosarios o guías de estilo ciertas cuestiones lingüísticas (léxico, aspectos gramaticales, estilo, etc.), que suelen inclinarse inevitablemente a una variedad determinada, mayoritariamente - con suerte, si el trabajo de preproducción se ha llevado a cabo correctamente - a la variedad del texto final.

Asimismo, otras herramientas elaboradas en preproducción, como las memorias de traduc-

Tabla 2. Modelo abreviado de proceso de traducción (adaptado de Cid-Leal et al., 2019, p. 204)

\begin{tabular}{c|cccc}
\hline PREPRODUCCIÓN & & & \\
ETAPA & $\begin{array}{c}\text { (elaboración de glo- } \\
\text { sarios, memorias de } \\
\text { traducción, traducción } \\
\text { automática, elaboración } \\
\text { de especificaciones de } \\
\text { proyecto, etc.) }\end{array}$ & $\begin{array}{c}\text { TRADUCCIÓN O } \\
\text { POSEDICIÓN }\end{array}$ & REVISIÓN & $\begin{array}{c}\text { CONTROL DE } \\
\text { CALIDAD O POS- } \\
\text { PRODUCCIÓN }\end{array}$ \\
\hline VARIEDAD & $\begin{array}{c}\text { SUPUESTA } \\
\text { SOLICITADA }\end{array}$ & VARIEDAD FINAL & $\begin{array}{c}\text { ESPAÑOL } \\
\text { NEUTRO }\end{array}$ & VARIEDAD \\
FINAL & VARIEDAD FINAL \\
\hline
\end{tabular}


470 ción y las traducciones automáticas para posedición, añaden un componente adicional que condiciona la variedad diatópica empleada por el traductor. Si la memoria del proyecto es apropiada — es decir, si la conforman segmentos de proyectos anteriores similares al presente y para la misma región - y la traducción automática (pretraducción) se ha procesado correctamente - con un procesamiento adecuado de recursos previos-, el resultado es que el texto pretraducido y las sugerencias de la/s memoria/s tienden irremediablemente hacia la variedad final. Dado que la tarea del poseditor «consiste esencialmente en corregir la traducción en bruto con el fin de obtener un texto que presente un nivel de calidad que ha sido determinado previamente» (Cid-Leal et al., 2019), se produce en este caso una notable incongruencia en las especificaciones del proyecto. Así, cuando un responsable de un proyecto de traducción solicita a un poseditor que corrija la pretraducción para obtener un español neutro que posteriormente va a modificarse a la variedad final, lo que realmente está pidiéndole es que invierta un esfuerzo tan inconmensurable como inútil en modificar un texto bruto ya instalado en la variedad final para luego deshacer ese trabajo de supuesta neutralización en el proceso de revisión.

De esta reflexión, cabe deducir que lo que realmente debería solicitarse no es que se aspire a un español neutro en la traducción o texto poseditado como paso intermedio, sino simplemente que el traductor/poseditor sea consciente de la variedad final y de sus limitaciones como no nativo en la elaboración de lo que se requiere como producto final. En otras palabras, que, si uno es español y el texto es para México, debe esforzarse por evitar introducir elementos que no pertenezcan a la variedad mexicana y, a la par, intentar no modificar elementos de la pretraducción o las sugerencias de la memoria que ya están en dicha variedad.
En consecuencia, estaríamos en este caso ante un uso impropio del término español neutro muy extendido entre empresas farmacéuticas y agencias de traducción que puede llevar a confusión a los traductores/poseditores.

\section{CONCLUSIONES}

De los apartados anteriores cabe extraer las siguientes conclusiones:

I. La celeridad y la globalización, notas características de la industria farmacéutica, hacen que los traductores de textos farmacéuticos tengan que enfrentarse con frecuencia a la práctica de traducir a variedades de las que no son nativos. Dicho esto, la práctica diaria de la traducción farmacéutica demuestra que las empresas farmacéuticas y las agencias de traducción que las asisten son mayoritariamente sensibles a las variedades diatópicas y emplean recursos en mantenerlas.

2. Las legislaciones de los países en materia de productos farmacéuticos constituyen un determinante fundamental de la variedad diatópica en traducción farmacéutica, puesto que la terminología farmacéutica oficial obligatoria no está consensuada y unificada entre los distintos países hispanohablantes. Por tanto, existen diferencias en terminología esencial que impiden que exista un único español farmacéutico. Además, estas diferencias son insalvables por el momento, puesto que un mismo término puede hacer referencia a distintos conceptos según la legislación del país en cuestión.

3. Existen herramientas, como el MedDRA y las DCI, que buscan alcanzar la unificación terminológica en el lenguaje farmacéutico, aunque estas herramientas podrían ser in- 
suficientes y, además, necesitarían de mayor respaldo internacional. Así, en muchos territorios (especialmente en Latinoamérica), el uso del MedDRA ni siquiera es obligatorio y hay otros países (como Puerto Rico, EE. UU.) que no emplean las DCI.

4. Dada la reglamentación nacional y europea existente, es plausible afirmar que existe la suficiente cohesión y unificación en el lenguaje farmacéutico del español de España como para aseverar que el español farmacéutico peninsular es lengua estándar para el conjunto de los españoles. Al contrario, dado el nivel de reglamentación menos estricto que el europeo y la multiplicidad de autoridades reguladoras en la región, no existe la suficiente cohesión y unificación del lenguaje farmacéutico del español de Latinoamérica como para afirmar que el español farmacéutico latinoamericano es lengua estándar para el conjunto de los latinoamericanos hispanohablantes. No obstante, sí pueden observarse en Latinoamérica tendencias lingüísticas generalizadas que divergen del español peninsular.

5. La conveniencia de un español neutro en un lenguaje especializado como el farmacéutico parece fuera de toda duda. Su existencia permitiría reducir los abultados costes de traducción del sector farmacéutico y cumplir con mayor holgura los reducidos plazos de presentación de traducciones con una sola versión española, sin necesidad de adaptación regional posterior. Sin embargo, dada la diversidad terminológica entre los países hispanohablantes mencionada en anteriores conclusiones, dicho supuesto español farmacéutico neutro se asemeja más a una entelequia que a la realidad, máxime dado que se trata del lenguaje de una ciencia experimental, con una génesis constante de neologismos que dificulta la homogeneización terminológica.

6. Por último, se evidencia en el sector de la traducción farmacéutica una carencia de reflexión profunda sobre la realidad del supuesto español farmacéutico neutro. Así, su empleo como paso intermedio en las cadenas de traducción/posedición parece más bien un uso impropio del término español neutro que puede llegar a confundir a los traductores.

\section{REFERENCIAS BIBLIOGRÁFICAS}

AEMPS (2010): «Presentación de traducciones de prospectos y fichas técnicas a la AEMPS: Indicaciones para su presentación y procedimiento para resolver traducciones pendientes», Agencia Española de Medicamentos y Productos Sanitarios - AEMPS, <https://www.aemps.gob.es/informa/notasInformativas/industria/2010/traduccion-ProspectosFT.htm>.

AEMPS (2016): «Guía para la correcta traducción de textos y elaboración de maquetas de medicamentos veterinarios», Agencia Española de Medicamentos y Productos Sanitarios - AEMPS, <https://www. aemps.gob.es/industria/regMedicamentos/docs/ guia_maqueta.pdf?x54046>.

NaVARro, Fernando A. (2020): «Medical device», en Fernando A. Navarro (ed.), Diccionario de dudas y dificultades de traducción del inglés médico (3. ${ }^{a}$ edición), España: Cosnautas.

Bravo García, Eva (2008): El español internacional: Conceptos, contextosy aplicaciones, Madrid: Arco Libros.

CABrÉ, María Teresa (2004): «Lenguajes especializados o lenguajes para propósitos específicos», Foro hispánico, 26, 19-23.

CABré, María Teresa y R. Estopà (2005): «Unidades de conocimiento especializado: caracterización y tipología», en M. T. Cabré y C. Bach (eds.), Coneixement, llenguatge $i$ discurs especialitzat, Barcelona: IULA, Documenta Universitaria, 69-95. 
472 CAStro, Xosé (2017): «El español neutro en la traducción», Blog @xosecastro, <http://xcastro. com/2017/10/05/espanol-neutro-traduccion/>.

Cid-Leal, Pilar; María Carmen Espín-García \& Marisa Presas (2019): «Traducción automática y posedición: Perfiles y competencias en los programas de formación de traductores», en Miguel ToLosA Igualada y Álvaro Echeverri (eds.): Porque algo tiene que cambiar. La formación de traductores e intérpretes: Presente \& future. Because something should change: Present \& Future Training of Translators and Interpreters. MonTI, 11, 187-214.

EDQM (2021): «Standard Terms», European Directorate for the Quality of Medicines \& Healthcare, $<$ https://standardterms.edqm.eu/>.

EMA (2021a): «Product-information templates - Human», European Medicines Agency. Science Medicines Health, <https:/www.ema.europa.eu/en/ human-regulatory/marketing-authorisation/ product-information/product-information-templates-human>.

EMA (2021b): «EudraVigilance system overview», European Medicines Agency. Science Medicines Health, $<$ https://www.ema.europa.eu/en/human-regulatory/research-development/pharmacovigilance/ eudravigilance/eudravigilance-system-overview>.

FernándeZ-OrdoñEZ, Inés (2016): «Dialectos del español peninsular», Javier GutiérREZ REXACH (ed.), Enciclopedia de Lingüística Hispánica, Reino Unido: Routledge.

FUNDEU (2011): «Hispanoamérica, Iberoamérica y Latinoamérica no son sinónimos», Fundéu - RAE, <https://www.fundeu.es/recomendacion/hispanoamerica-iberoamerica-latinoamerica/\#: :text=Su\%20gentilicio\%20 s\%20latinoamericano., habla\%20 portuguesa\%2C\%20el\%20t\%C3\%A9rmino\%20 Iberoam\%C3\%A9rica>.

GARCÍA IZQUIERDO, Isabel (2006a): «El español neutro y la traducción de los lenguajes de especialidad», Sendebar, 17, 149-167.

GARCíA IzQUIERDo, Isabel (2006b): «Neutral Spanish, Spanglish and Medical Translation. A case of heterodoxy», Translation Journal, 10 (3), <https://translationjournal.net/journal/37neutro.htm>.
GARgATAGLI, Marietta (2012): «La traducción neutra no es una pipa», Blog Ciudad de las Ideas, <http:// ciudadideas.blogspot.com/2012/10/la-traduccion-neutra-no-es-una-pipa.html>.

Gómez Font, A. (2012): «Español neutro, global, general, estándar o internacional», Ómnibus, 39.

ICH (2020): «Members \& Observers», ICH, <https:// www.ich.org/page/members-observers $>$.

La Prensa (2010): «Los hispanohablantes de América prefieren el gentilicio "latinoamericano", según encuesta», La Prensa, <https://www.laprensa.com. ni/2010/05/25/internacionales/25668-los-hispanohablantes-de-america-prefieren-el-gentiliciolatinoamericano-segun-encuesta>.

López Morales, Humberto (2006): La globalización del léxico hispánico, Madrid: ESPASA FÓRUM.

MARCILly, Christian R. et al. (2020): «How to interact with medical terminologies? Formative usability evaluations comparing three approaches for supporting the use of MedDRA by pharmacovigilance specialists», BMC Medical Informatics and Decision Making, 20 (1), 261-275, <https://zaguan.unizar.es/ record/96111? $\ln =\mathrm{es}>$.

RAE (2005): «Diccionario panhispánico de dudas», Real Academia Española, <https://www.rae.es/ $\mathrm{dpd} />$.

RAmírez Luengo, José Luis (2011): «Imaginar lo imposible: Algunas reflexiones sobre el denominado español neutro», en Daniel M. SÁEz Rivera, Jorge Braga Riera, Marta Abuín González, Marta Guirao Ochoa, Beatriz Soto Aranda y Nava Maroto GARCía (eds.), Últimas tendencias en traducción e interpretación, Madrid: Editorial Iberoamericana, 17-24.

RED EAMI (n. d.): «Directorio de autoridades», Red de Autoridades en Medicamentos de Iberoamérica, <https://www.redeami.net/web/eami/seccion/ contenedor_secciones/eami_conten_directorio_ de_autoridades.htm $>$.

RodrígueZ-Perdomo, Tenesor (2012): «Promotor o patrocinador: las apariencias importan», Panace@, 13 (36), 346.

SÁENZ, Miguel (2013): «Traducir literatura al español, pero ¿a qué español?», Dieciocho conferencias nada 
magistrales $y$ dos discursos de circunstancias, en Miguel SÁEnz (ed.), España: Ediciones Universidad de Salamanca, 81-92.

SocA, Ricardo (2010): «¿Hispanoamericanos o latinoamericanos?», elcastellano.org, <https://www. elcastellano.org/\%C2\%BFhispanoamericanos-olatinoamericanos $>$.

Villalba, Gabriela (2017): «Representaciones sobre el español en la traducción editorial argentina: metodología de una investigación», El taco en la brea, 5, 380-407.

Zaro Vera, Juan Jesús (2013): «La traducción, “zona de conflicto” entre España y la América hispana», Revista Debats, 121, 74-83. 